\title{
Experimental Validation of Theoretical Models for the Linear and Nonlinear Vibrations of Immersed Rotors
}

\author{
M. MOREIRA ${ }^{1}$, A. TISSOT ${ }^{2}$, and J. ANTUNES ${ }^{3, *}$ \\ ${ }^{1}$ Polytechnic Institute of Setubal, Portugal; ${ }^{2}$ Institute of Nuclear Technology; ${ }^{3}$ Institute of Nuclear Technology
}

\begin{abstract}
Vibration of rotating shafts has been studied for different gap geometries, ranging from bearing configurations to pump systems. This paper deals with the rotor-flow dynamics of immersed shafts under moderate confinement - clearance gap about $\delta=H / R=0.1$ (where $H$ is the average gap and $R$ is the rotor radius). Following simplified assumptions, analytical models for the linearized forces, for both centered and eccentric immersed rotors have been developed as well as a theoretical nonlinear model which fully describes the nonlinear flow terms. These models were supported by encouraging results from preliminary experiments. In the present paper, we discuss some recent and representative results of an extensive series of tests performed on a small-scale model, in order to assert the validity of our theoretical models.

From the overall experimental programma, the following conclusions emerged: (1) The linearized bulk-flow model is adequate, provided the dissipative effects are duly accounted for using an empirical friction coefficient to empirically model the turbulent stresses. Such predictions are quite accurate if the system is working at low rotor eccentricities and far from the instability boundaries. (2) However, for large rotor eccentricities and for dynamic regimes near the linear instability, the fully nonlinear model leads to better predictions. Obviously, these effects are instrumental to obtaining reasonable predictions for all post-stable motion regimes. (3) When discrepancies arise, the nonlinear model was usually found to be conservative.
\end{abstract}

Keywords: Rotor vibrations; Nonlinear dynamics; Flow-structure interaction; Experimental identification

Received 23 May 2000; In final form 21 June 2000

*Corresponding author. Tel.: 351219946 00, Fax: 351219550117. E-mail: jantunes@itn1.itn.pt
The effects of co-rotating annular flows on rotor vibrations have been studied extensively by many researchers, mostly in connection with bearings and seals (see for instance Childs, 1993). However, flow structure interaction can also lead to significant effects in less common devices. In this paper we will address the dynamical behavior of immersed rotors, such as found in fast-breeder nuclear reactor pumps (for circulating the liquid sodium), and other applications. In such components, the clearance ratio $\delta=H / R$ (where $H$ is the average gap and $R$ is the rotor radius) is typically about 0.1 - one or two orders of magnitude higher than the clearances found in bearings and seals. As a consequence, the flow is quite turbulent, inertial effects are then of prime importance and cannot be neglected as assumed in the basic Reynolds equation approach. Also, the shaft length subject to fluid forces is quite significant, these combined effects leading to specific rotor-dynamic properties.

Using simplified assumptions for the flow, we have developed analytical models for the linearized forces, for both centered and eccentric rotors (Axisa \& Antunes, 1992; Antunes et al., 1996 and Moreira et al., 2000). Because the flow was modeled as being two-dimensional, it was possible to extend our analytical solutions to fully account for the nonlinear flow terms (Antunes et al., 1999 and Moreira et al., 1999), in contrast with most numerical solutions found in other papers.

\section{EXPERIMENTAL SETUP}

Experiments were performed using a rigid rotor supported by a flexible support. Two flexible support configurations were used, in order to study both planar motions - motions $X(t)$ taking place in one single direction - and orbital motions - motions $X(t)$ and $Y(t)$ taking place in orthogonal directions. The test rig consists of a vertical shaft with radius $R=25 \mathrm{~mm}$ and length $L=365 \mathrm{~mm}$. The shaft is mounted 


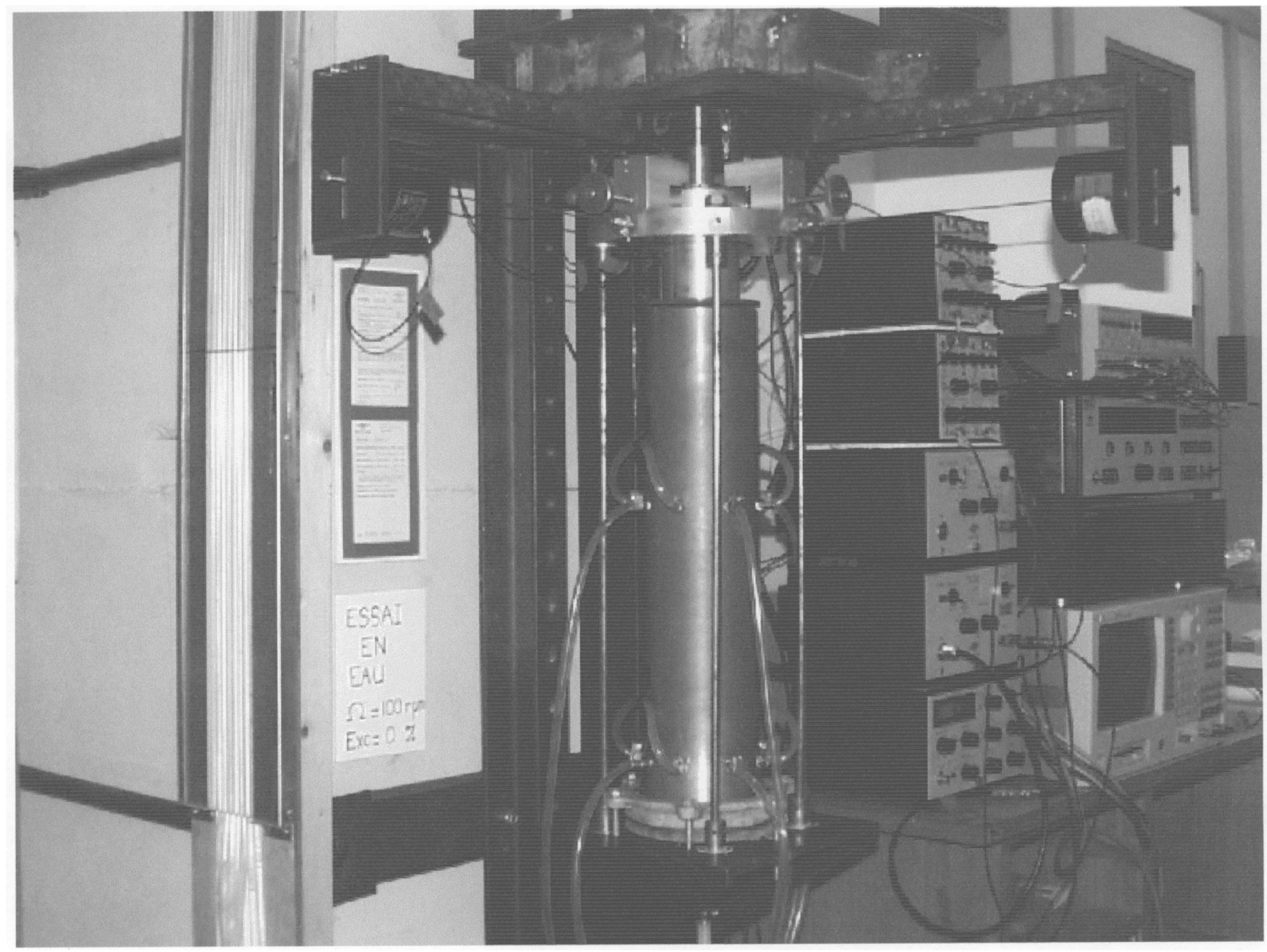

FIGURE 1 Orbital setup.

using a pair of conical bearings, assembled in a rigid support plate held in position by four flexible struts. Figure 1, shows the setup for orbital motions, where symmetrical flexible shaft supports provide $K_{s}^{Y}=K_{s}^{X}$. Planar motions were insured by using asymmetric supports (with rectangular cross-section) providing $K_{s}^{Y} \gg K_{s}^{X}$, as shown in Figure 2.

The shaft rotates inside a rigid outer shell, the full length of the annular gap being filled with water. The average gap was $H=2 \mathrm{~mm}$ and shaft-to-shell eccentricity could be varied in the range $\varepsilon=0 \sim 100 \%$. The shaft was driven by an electric motor with precise velocity control, using a double cardan coupling for torque transmission. To avoid surface effects and flow ventilation at higher spinning velocities, a large-clearance labyrinth was used. Rotor vibrations were measured with eddy-current displacement transducers, located near the support plate. Excitation in planar experiments was provided by a non-contact electromechanical shaker and, in orbital experiments, by two electro-mechanical shakers, driven by filtered random noise. The excitation force was measured using piezoelectric transducers. Accelerometers were also used in order to perform an accurate sensor calibration and improve measurement accuracy. In Figure 2, some details of sensor setup can be observed (planar experiments). The modal parameters of the coupled system and the non-linear dominant response frequencies and damping were identified using the ERA method-Eigensystem Realization Algorithm (Juan, 1994).

The main physical parameters of the test rig are shown in Table I. The experimental modal parameters of the two planar and the two orbital tested configurations, which are discussed here, were labeled respectively $\mathrm{P} 1, \mathrm{P} 2, \mathrm{O} 1$ and $\mathrm{O} 2$. The corresponding parameters can be seen in Tables II and III.

\section{THEORETICAL PREDICTIONS}

For the complex modal computations of the rotor-flow coupled system, the improved linear model (deduced from the linearized forms of Eq. [8]) for rotors subject to dissipative annular flows developed by Moreira et al., 2000, was used. In addition to the forward and backward whirling modes predicted by the classic linear theory the new improved model also presents a zero-frequency eigenvalue related to the co-rotating flow.

Nonlinear numerical simulations were based in the model for nonlinear orbital motions, developed by Moreira et al., 


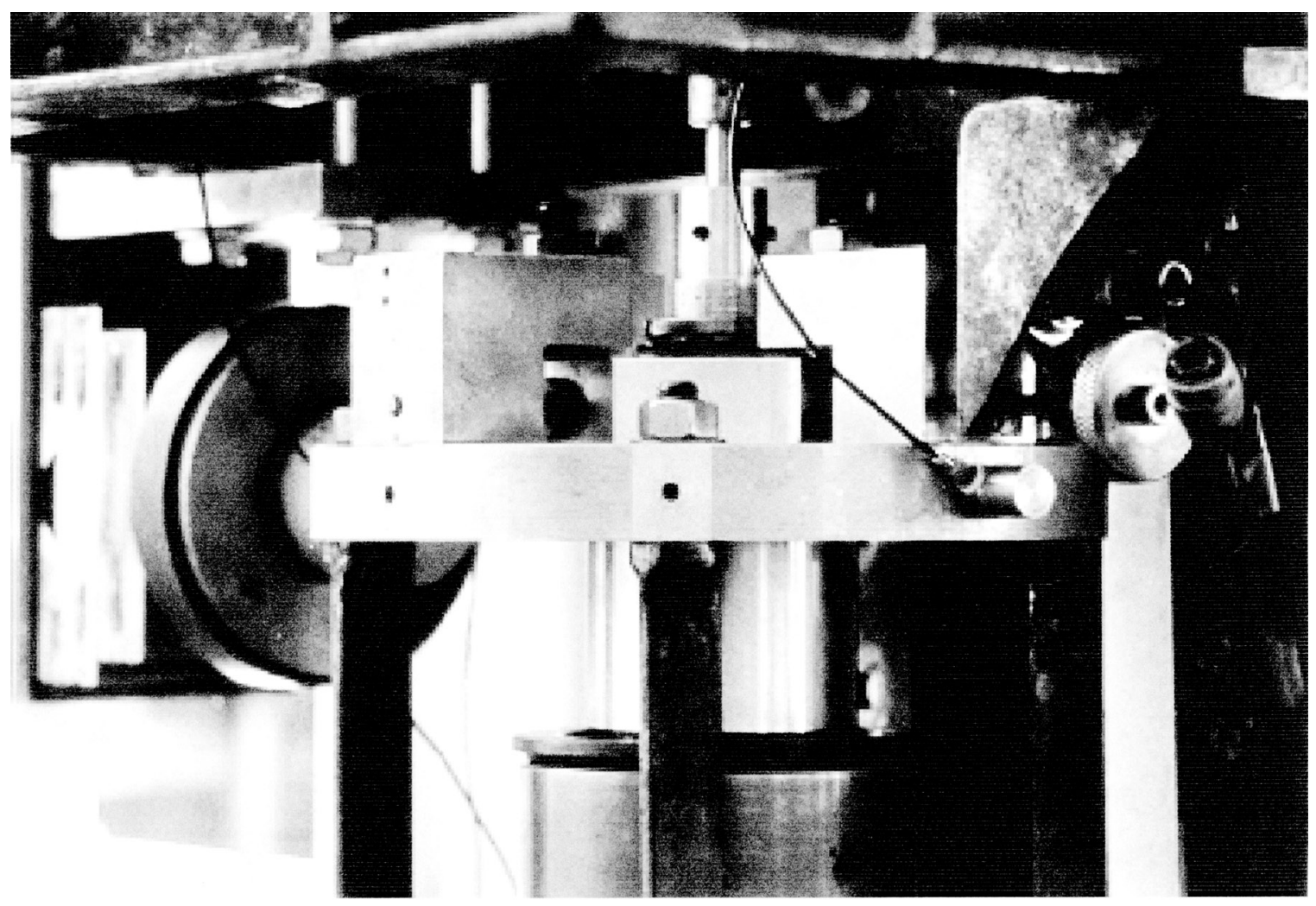

FIGURE 2 Planar setup details.

TABLE I Main physical parameters

\begin{tabular}{ll}
\hline Rotor length, $L(\mathrm{~m})$ & 0.365 \\
Rotor radius, $R(\mathrm{~m})$ & 0.0251 \\
Annular gap, $H(\mathrm{~m})$ & 0.002 \\
Clearance ratio, $\delta=H / R$ & 0.08 \\
Length-to-diameter ratio, $L / D$ & 7.3 \\
\hline
\end{tabular}

TABLE II Physical and modal parameters of the planar experiments

\begin{tabular}{lcc}
\hline & $\mathrm{P} 1$ & $\mathrm{P} 2$ \\
Eccentricity, $\varepsilon$ & 0 & 0.5 \\
\hline Structural mass, $M_{s}(\mathrm{~kg})$ & 7.7 & 7.7 \\
Mass ratio $\gamma=M_{a} / M_{s}$ & 1.2 & 1.3 \\
$X$ str. stiff., $K_{s}^{X}\left((\mathrm{~N} / \mathrm{m}) / 10^{4}\right)$ & 1.7 & 1.7 \\
$Y$ str. stiff., $K_{s}^{Y}\left((\mathrm{~N} / \mathrm{m}) / 10^{4}\right)$ & $K_{s}^{Y} \gg K_{s}^{X}$ & $K_{s}^{Y} \gg K$ \\
Freq. in air $(\mathrm{Hz})$ & 7.5 & 7.5 \\
Freq. in water $(\mathrm{Hz})$ & 5.1 & 5.0 \\
Damping in air $(\%)$ & 0.4 & 0.4 \\
Damping in water $(\%)$ & 3.7 & 5.0 \\
\hline
\end{tabular}

1999. The rotodynamic nonlinear equations for this model, are as follows

$$
\begin{gathered}
\ddot{X}=\mathbb{F}_{1}\left[\dot{X}, X, \dot{Y}, Y, C, F_{X}^{\mathrm{ext}}, t\right] \\
\ddot{Y}=\mathbb{F}_{2}\left[\dot{X}, X, \dot{Y}, Y, C, F_{Y}^{\mathrm{ext}}, t\right], \\
\dot{C}=\mathbb{F}_{3}\left[\ddot{X}, \dot{X}, X, \ddot{Y}, \dot{Y}, Y, C, F_{X}^{\mathrm{ext}}, F_{Y}^{\mathrm{ext}}, t\right],
\end{gathered}
$$

TABLE III Physical and modal parameters of the experiments

\begin{tabular}{lll}
\hline & $\mathrm{O} 1$ & $\mathrm{O} 2$ \\
Eccentricity, $\varepsilon$ & 0 & 0.5 \\
\hline Structural mass, $M_{s}(\mathrm{~kg})$ & 7.1 & 7.1 \\
Mass ratio $\gamma=M_{a} / M_{s}$ & 1.3 & 1.4 \\
$X$ str. stiff., $K_{s}^{X}\left((\mathrm{~N} / \mathrm{m}) / 10^{4}\right)$ & 1.16 & 1.16 \\
$Y$ str. stiff., $K_{s}^{Y}\left((\mathrm{~N} / \mathrm{m}) / 10^{4}\right)$ & 1.16 & 1.16 \\
Freq. in air $(\mathrm{Hz})$ & 6.5 & 6.5 \\
Freq. in water $(\mathrm{Hz})$ & 4.5 & 4.4 \\
Damping in air $(\%)$ & 0.5 & 0.5 \\
Damping in water $(\%)$ & 4.0 & 4.1 \\
\hline
\end{tabular}

where $X, Y$ define the rotor motion, $C=C(t)$ is an auxiliary flow variable, $F_{X}^{\mathrm{ext}}, F_{Y}^{\mathrm{ext}}$ represent autonomous forces and $t$ time. The nonlinear formulation, for the fluidelastic forces $F_{X}(t)$ and $F_{Y}(t)$ (and an additional auxiliary equation) which lead to Eqs. [1]-[3] was deduced (under simplifying assumptions) from the continuity equation for incompressible flow and the momentum equation (projected in the tangential direction),

$$
\begin{gathered}
0=\frac{\partial h}{\partial t}+\frac{1}{R} \frac{\partial(h u)}{\partial \theta} \\
0=\rho\left\{\frac{\partial(h u)}{\partial t}+\frac{1}{R} \frac{\partial\left(h u^{2}\right)}{\partial \theta}\right\}+\tau_{s}+\tau_{r}+\frac{h}{R} \frac{\partial p}{\partial \theta},
\end{gathered}
$$


where $h$ is the annular gap depth

$$
h(\theta, t)=H-X(t) \cos \theta-Y(t) \sin \theta,
$$

$\rho$ is the fluid density, $p$ is the gap-averaged pressure and $\tau_{s}$ and $\tau_{r}$ the shear stresses at the rotor and stator walls.

After obtaining the velocity field from Eq. [4]

$$
u(\theta, t)=\frac{R(\dot{X}(t) \sin \theta-\dot{Y}(t) \cos \theta+C(t))}{H-X(t) \cos \theta-Y(t) \sin \theta}
$$

the fluidelastic forces $F_{X}(t)$ and $F_{Y}(t)$ (and the additional auxiliary equation) can be deduced from Eq. [5]:

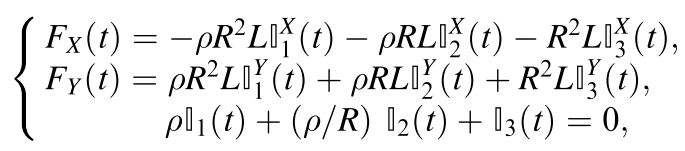

where $\square_{1}^{X}(t), \square_{2}^{X}(t), \square_{3}^{X}(t), \square_{1}^{Y}(t), \square_{2}^{Y}(t), \square_{3}^{Y}(t), \square_{1}(t), \square_{2}(t)$ and $\square_{3}(t)$ depend on the following azimuthal integrals

$$
G_{k}^{i j}(H, X, Y)=\int_{0}^{2 \pi} \frac{\sin ^{i} \theta \cos ^{j} \theta}{(H-X \cos \theta-Y \sin \theta)^{k}} d \theta
$$

for $0 \leq i, j, k \leq 4$.

All details of these theoretical formulations are presented in Moreira et al., 1999 and Moreira et al., 2000.

\section{RESULTS AND DISCUSSION}

The main results of our tests and computations are resumed in several figures which show the modal frequencies and damping values of the system as a function of the rotor eccentricity and spinning velocity. In these figures we display the real part $\sigma_{n}$ as well as the imaginary part $\omega_{n}$ of the complex eigenvalues $\lambda_{n}=\sigma_{n}+i \omega_{n}$ of the linearized model. Also the corresponding identified response frequencies and damping values from experiments and numerical simulations are displayed.

The predicted modal behavior of eccentric planar and orbital configurations $\mathrm{P} 2$ and $\mathrm{O} 2$ are illustrated in Figures 3 and 4.

In addition to the forward and backward whirling modes, there is a zero-frequency mode, related to the corotating flow, which can be identified in the figures exhibiting a non zero damping. The computed modes in Figures 3 and 4 are identified in the plots using the following codes: Planar configurations $-\mathrm{V}$ for the vibrating mode and $\mathrm{Z}$ for the zero-frequency eigenvalue; Orbital configurations - F and B respectively for the forward and
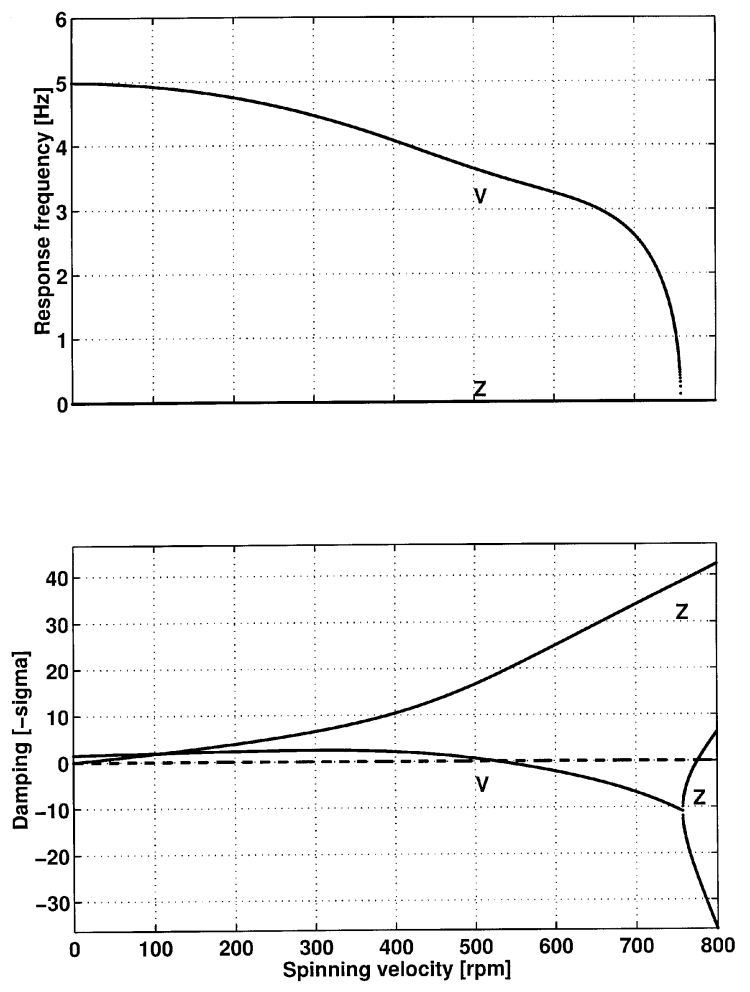

FIGURE 3 Planar configuration P2: $\varepsilon=0.5$, predicted modal behavior.
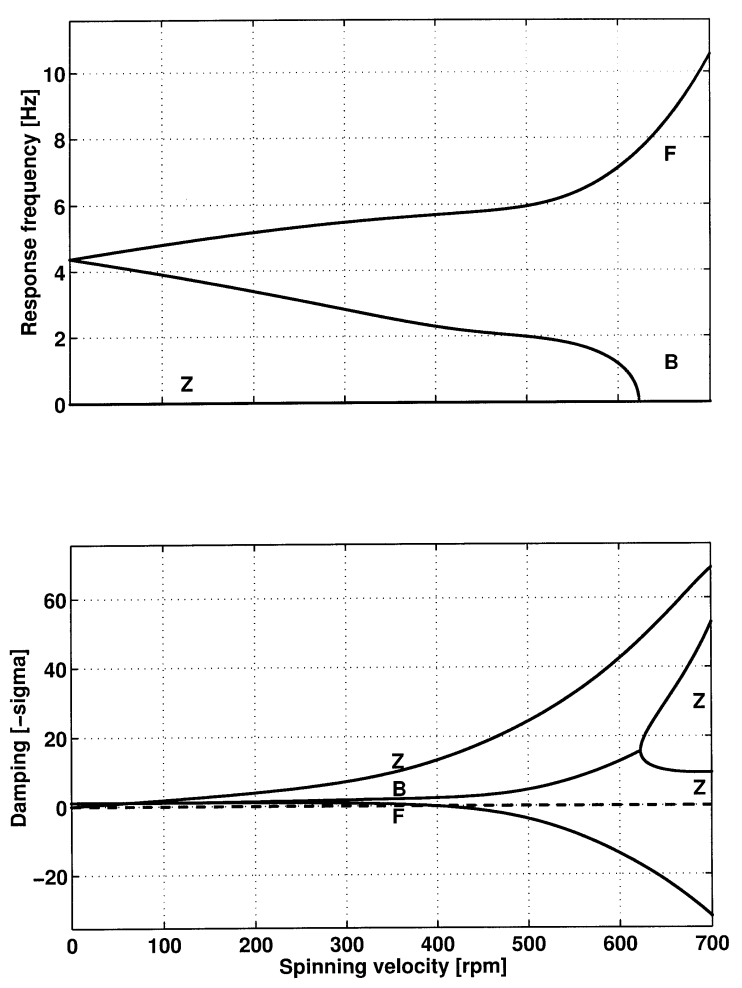

FIGURE 4 Orbital configuration $\mathrm{O} 2: \varepsilon=0.5$, predicted modal behavior. 
backward whirling modes and $\mathrm{Z}$ for the zero-frequency mode.

Note that, because system dynamics are strongly dependent on the rotor eccentricity, the linearized predictions of rotor dynamics, were computed using an estimate of the actual eccentricity at each spinning velocity, which was obtained from nonlinear simulations. This estimate proved to be accurate as confirmed by the results shown in Figure 5.
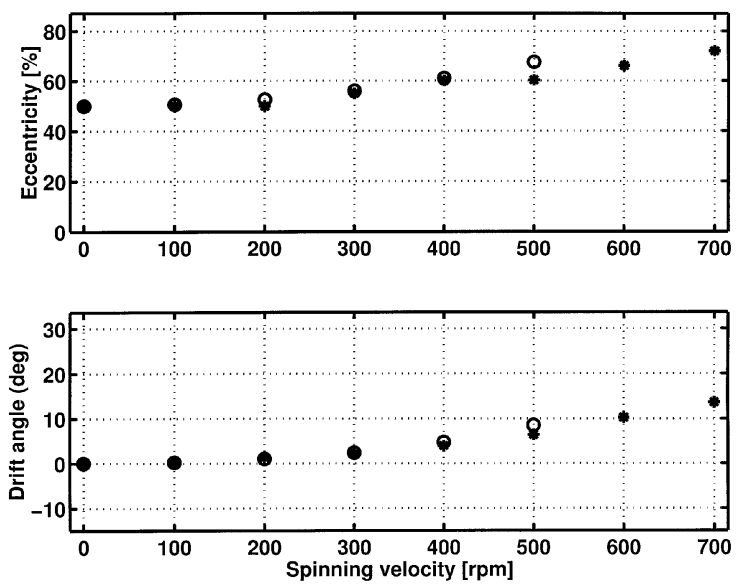

FIGURE 5 Orbital configuration O2: $\varepsilon=0.5$, magnitude and angle of the steady drift ( $\circ$, nonlinear simulations; $*$, experiments).
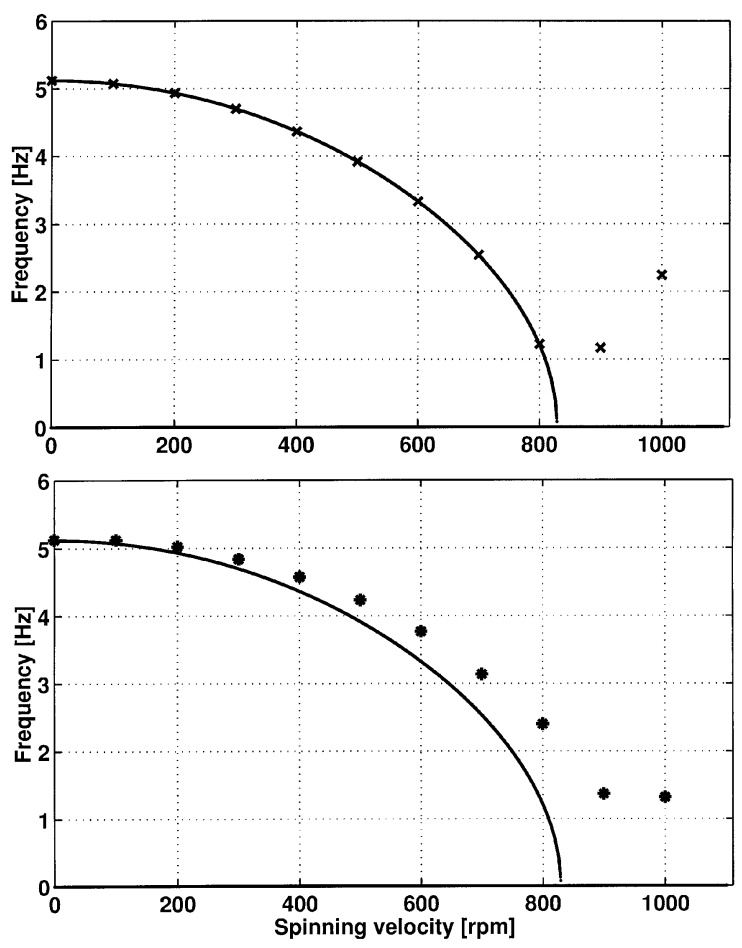

FIGURE 6 Comparison of modal frequencies for planar configuration $\mathrm{P} 1: \varepsilon=0$ (-, linear theory; $\times$, nonlinear simulations; $*$, experiments).
The main theoretical and experimental results of this paper are summarized in Figures 6-9 (planar tests) and Figures $10-13$ (orbital tests).
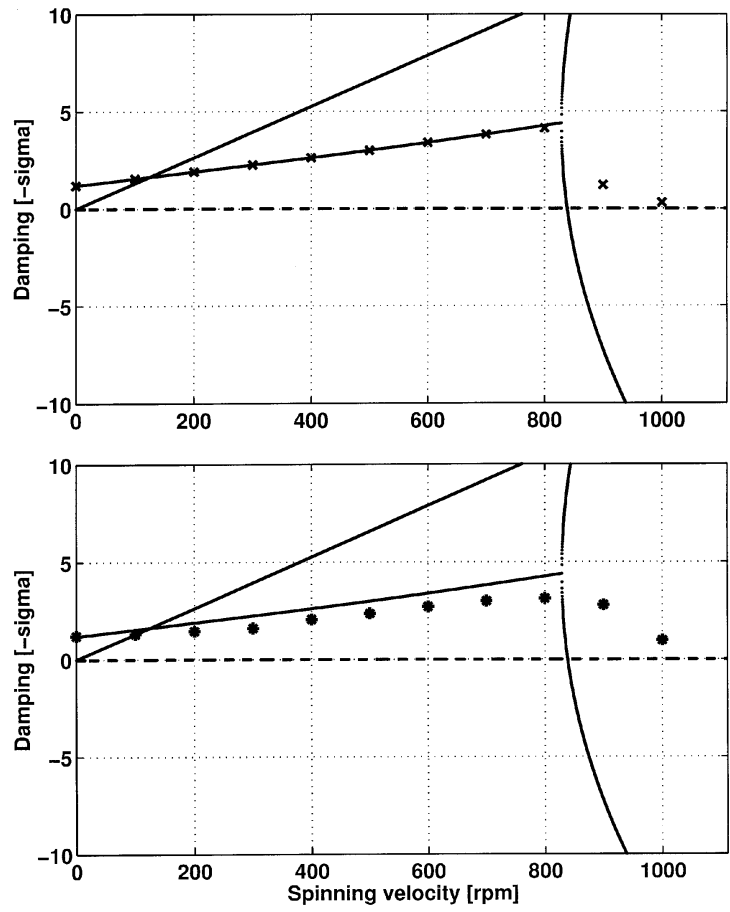

FIGURE 7 Comparison of modal damping for planar configuration P1: $\varepsilon=0$ (-, linear theory; $\times$, nonlinear simulations; $*$, experiments).
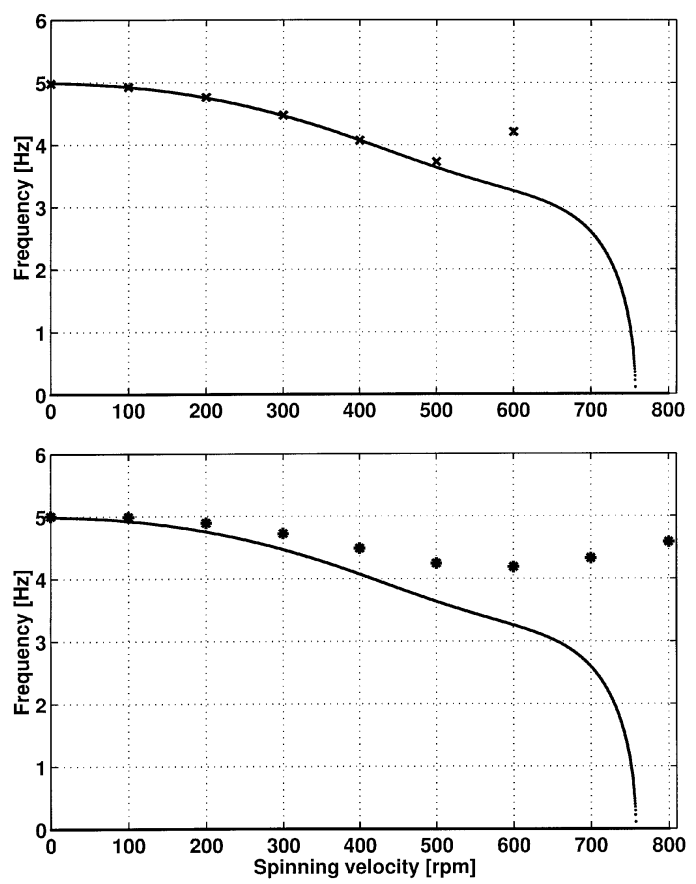

FIGURE 8 Comparison of modal frequencies for planar configuration P2: $\varepsilon=0.5$ (-, linear theory; $\times$, nonlinear simulations; $*$, experiments). 

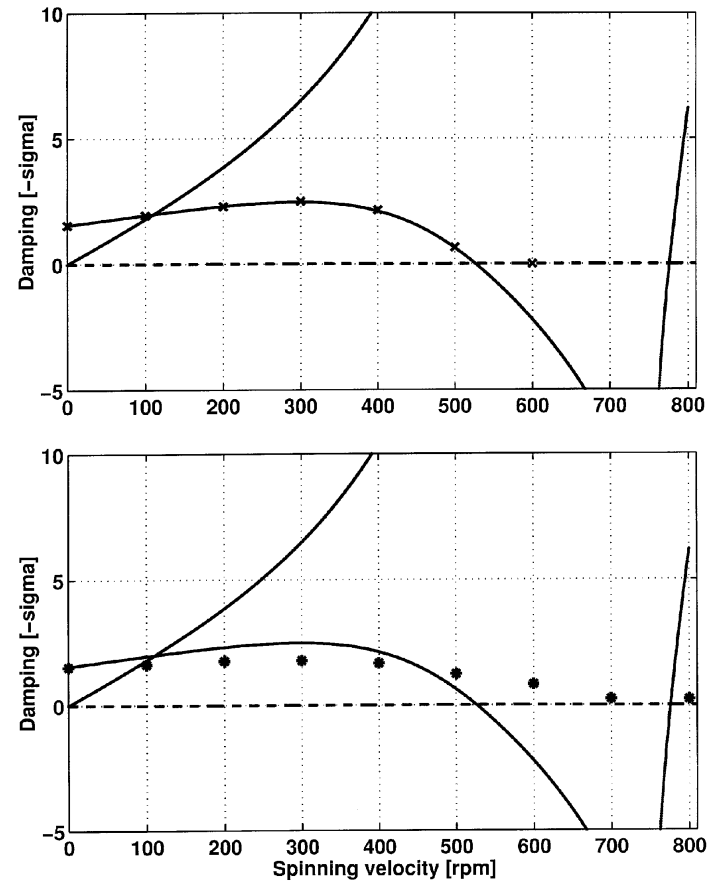

FIGURE 9 Comparison of modal damping for planar configuration P2: $\varepsilon=0.5$ (-, linear theory; $\times$, nonlinear simulations; $*$, experiments).
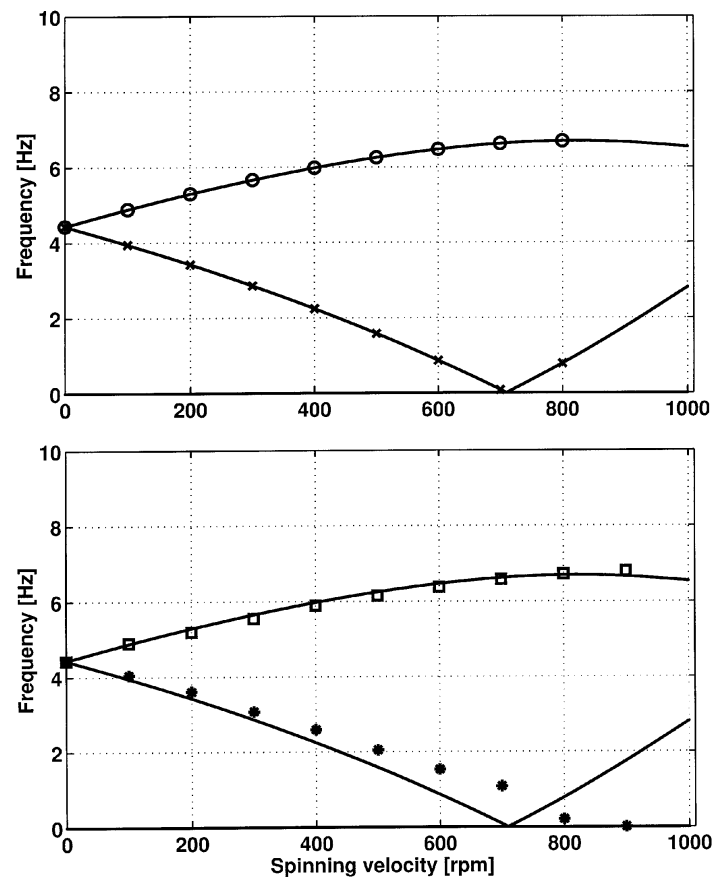

FIGURE 10 Comparison of modal frequencies for orbital configuration $\mathrm{O} 1: \varepsilon=0(-$, linear theory; $\circ$, first "mode" -nonlinear numerical simulations; $\times$, second "mode" - nonlinear numerical simulations; $\square$, first "mode"-experiments; $*$, second "mode"-experiments).
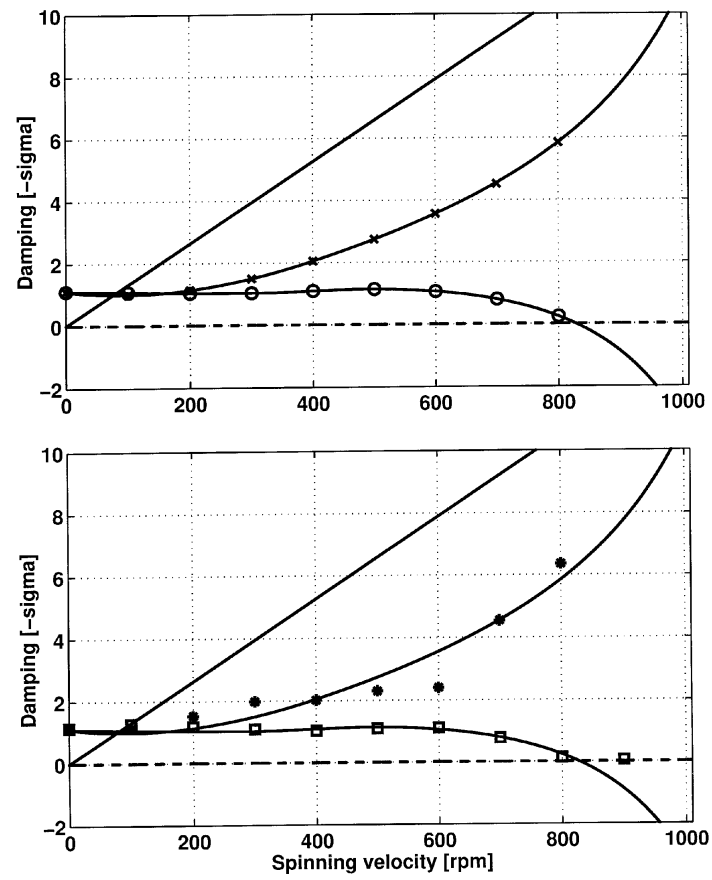

FIGURE 11 Comparison of modal damping for orbital configuration $\mathrm{O} 1: \varepsilon=0(-$, linear theory; $\circ$, first "mode" -nonlinear numerical simulations; $\times$, second "mode" - nonlinear numerical simulations; $\square$, first "mode"-experiments; $*$, second "mode"-experiments).
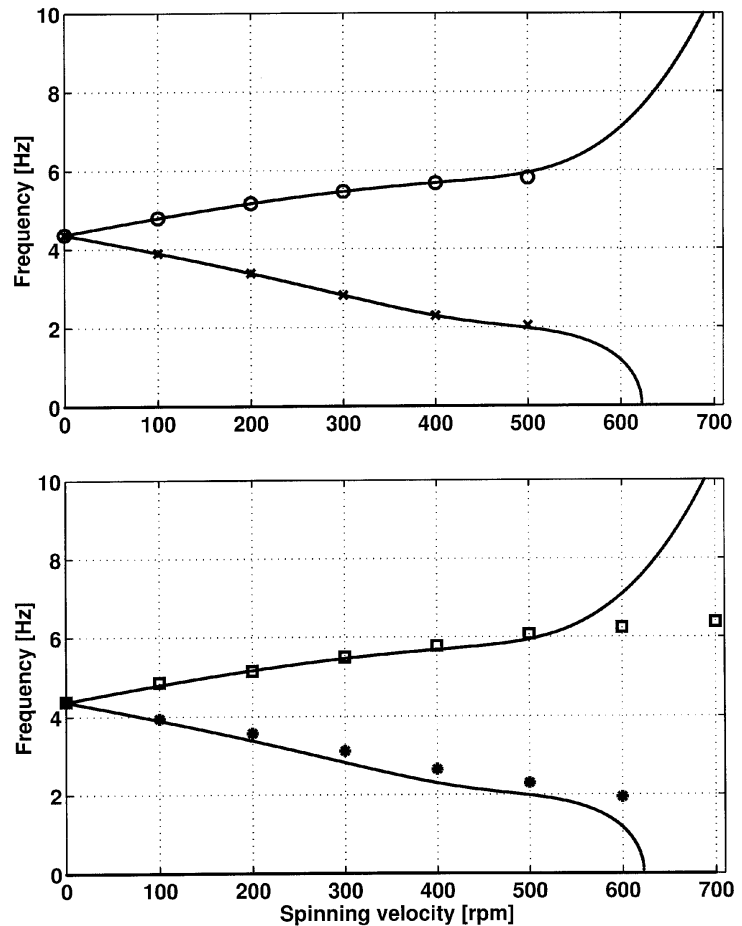

FIGURE 12 Comparison of modal frequencies for orbital configuration $\mathrm{O} 2: \varepsilon=0.5$ (-, linear theory; $\circ$, first "mode" - nonlinear numerical simulations; $\times$, second "mode" - nonlinear numerical simulations; $\square$, first "mode"-experiments; $*$, second "mode"-experiments). 

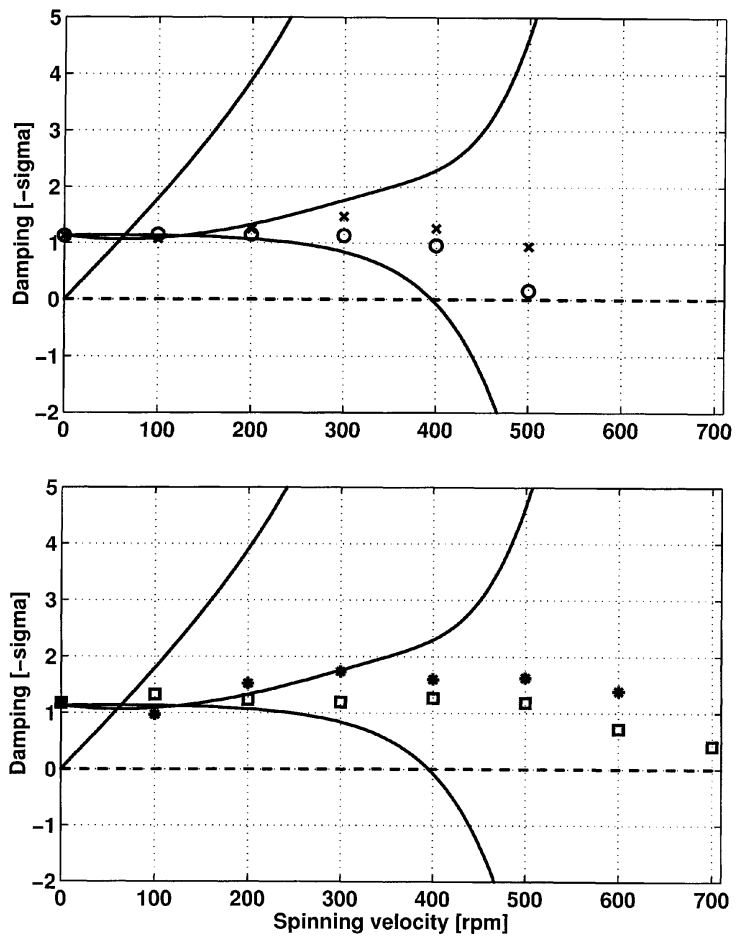

FIGURE 13 Comparison of modal damping for orbital configuration $\mathrm{O} 2: \varepsilon=0.5$ (-, linear theory; $\circ$, first "mode" - nonlinear numerical simulations; $x$, second "mode" - nonlinear numerical simulations; $\square$, first "mode"-experiments; *, second "mode"-experiments).

\section{Planar Configurations}

In Figures 6-9 one can observe the identified response frequencies and damping values which were identified from the experiments and nonlinear simulations. These are superimposed to the modal frequencies and damping predictions stemming from the linear theory, for both centered and eccentric planar configurations $\mathrm{P} 1$ and $\mathrm{P} 2$.

In planar centered configuration $\mathrm{P} 1$, linear and nonlinear theory agree very well with experiments below the predicted instability onset. In this range the dynamical behavior of the system is linear. Linear instability is predicted at about $820 \mathrm{rpm}$. Above this range and below $1000 \mathrm{rpm}$ the system becomes linearly unstable and so only the nonlinear simulations yields results which are similar to experiments. In Figure 14 one can detect a deterioration of the values displayed by the coherence function (in experiments) above $800 \mathrm{rpm}$. Post-stable motions are very well predicted by the nonlinear theory.

In planar configuration P2 (static reduced eccentricity equal to 0.5 ) linear theory predicts instability by flutter at about $520 \mathrm{rpm}$, which is a lower value than obtained from both the experiments and nonlinear theory. Indeed high-amplitude motions were obtained only at about $600 \mathrm{rpm}$ (nonlinear numerical simulations) and $800 \mathrm{rpm}$ (experiments).
From Figure 8 one can notice that the system vibrating frequency depart from linear predictions at about $500 \mathrm{rpm}$ confirming the significance of the nonlinear effects. Therefore, we have found that nonlinear flow forces are very significant for the eccentric configuration, even at relatively low spinning velocities. This is attested by the low values of the coherence function (in experiments) displayed in Figure 15.

\section{Orbital Configurations}

The identified response frequencies and damping from experiments and nonlinear simulations, for the orbital configurations $\mathrm{O} 1$ and $\mathrm{O} 2$ as well as the modal frequencies and damping values from the linear theory, are shown in Figures $10-13$.

In the orbital centered configuration $\mathrm{O} 1$, linear and nonlinear formulations agree very well below $600 \mathrm{rpm}$. In this range both formulations give acceptable predictions of the experimental results. Indeed the system behavior is essentially linear, as confirmed by the high values of the coherence function shown in Figure 16. These plots were computed using the system responses obtained from the numerical simulations of the nonlinear flow model.

Flutter instability is predicted at about $800 \mathrm{rpm}$ by both linear and nonlinear formulations. Indeed, large amplitude motions were experimentally observed at about $900 \mathrm{rpm}$. It is clear from these results that this centered configuration is mostly controlled by the linear fluid terms, up to the onset of flutter instability.

In the eccentric orbital configuration $\mathrm{O} 2$ the linearized and nonlinear formulations agree only up to $300 \mathrm{rpm}$. In Figure 17 one can observe high coherence values displayed below $400 \mathrm{rpm}$. Beyond this range, as in the planar case, nonlinear effects become significant. Indeed, the linearized predictions of damping values fail for all spinning velocities higher than $400 \mathrm{rpm}$.

Both experiments and nonlinear computations agree that the post-stable nonlinear system will display high-amplitude forward whirling orbits, for this rig. Also, linear instability is predicted for the forward mode at about $400 \mathrm{rpm}$. The agreement between the nonlinear model and experiments is thus quite satisfactory. However, as in the case for centered configuration $\mathrm{O} 1$, the predicted onset for high-amplitude motions is somewhat conservative.

We suspect that such behavior might be due to threedimensional flow end-effects, related to the relatively low $L / D$ ratio of our experimental rig. Indeed, the simplified two-dimensional flow model used for our predictions is inadequate if three-dimensional flow effects are significant. At the present time it is difficult to quantify the magnitude of such effects. 

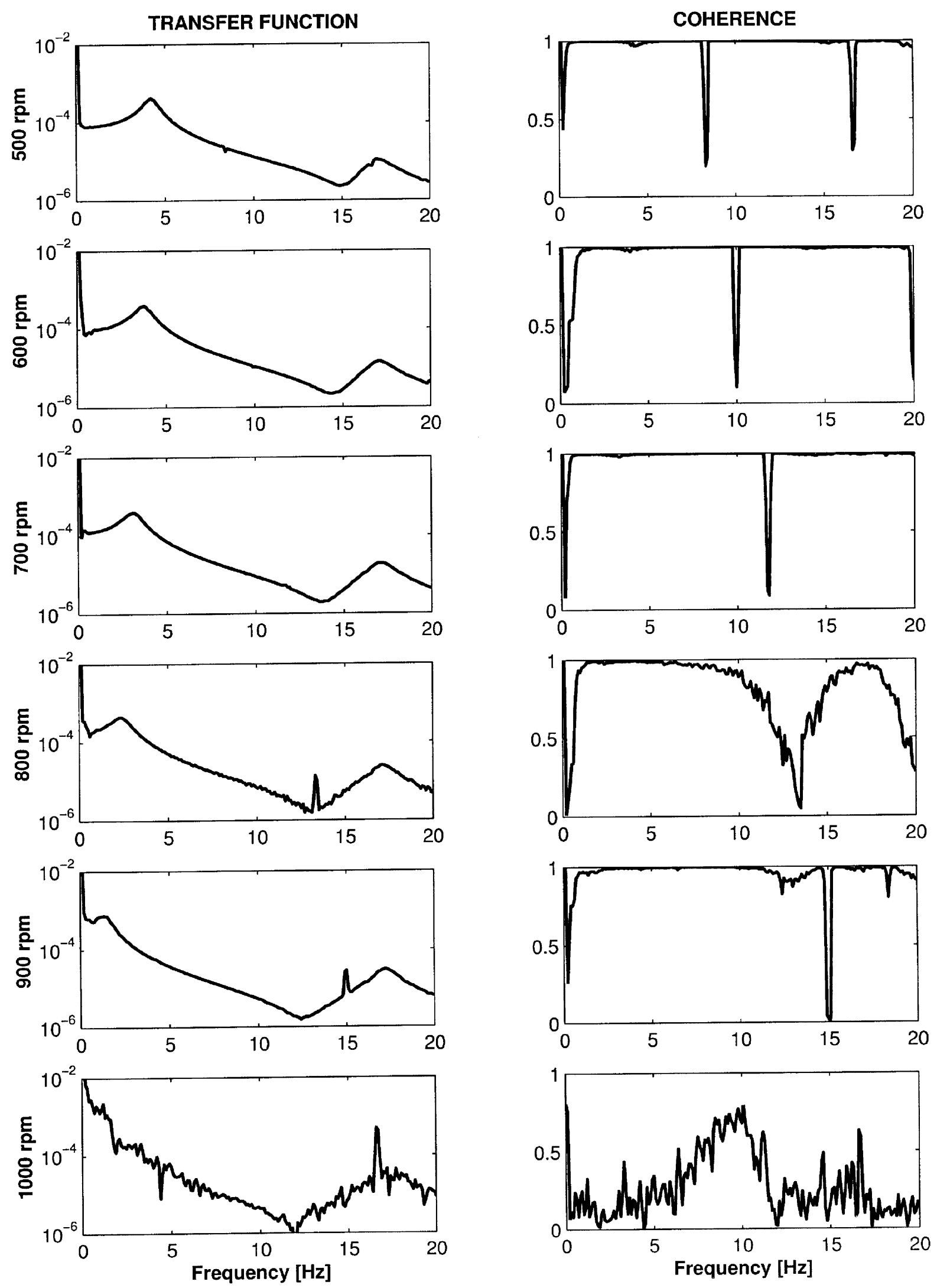

FIGURE 14 Planar configuration P1: $\varepsilon=0$, transfer and coherence functions (experiments). 

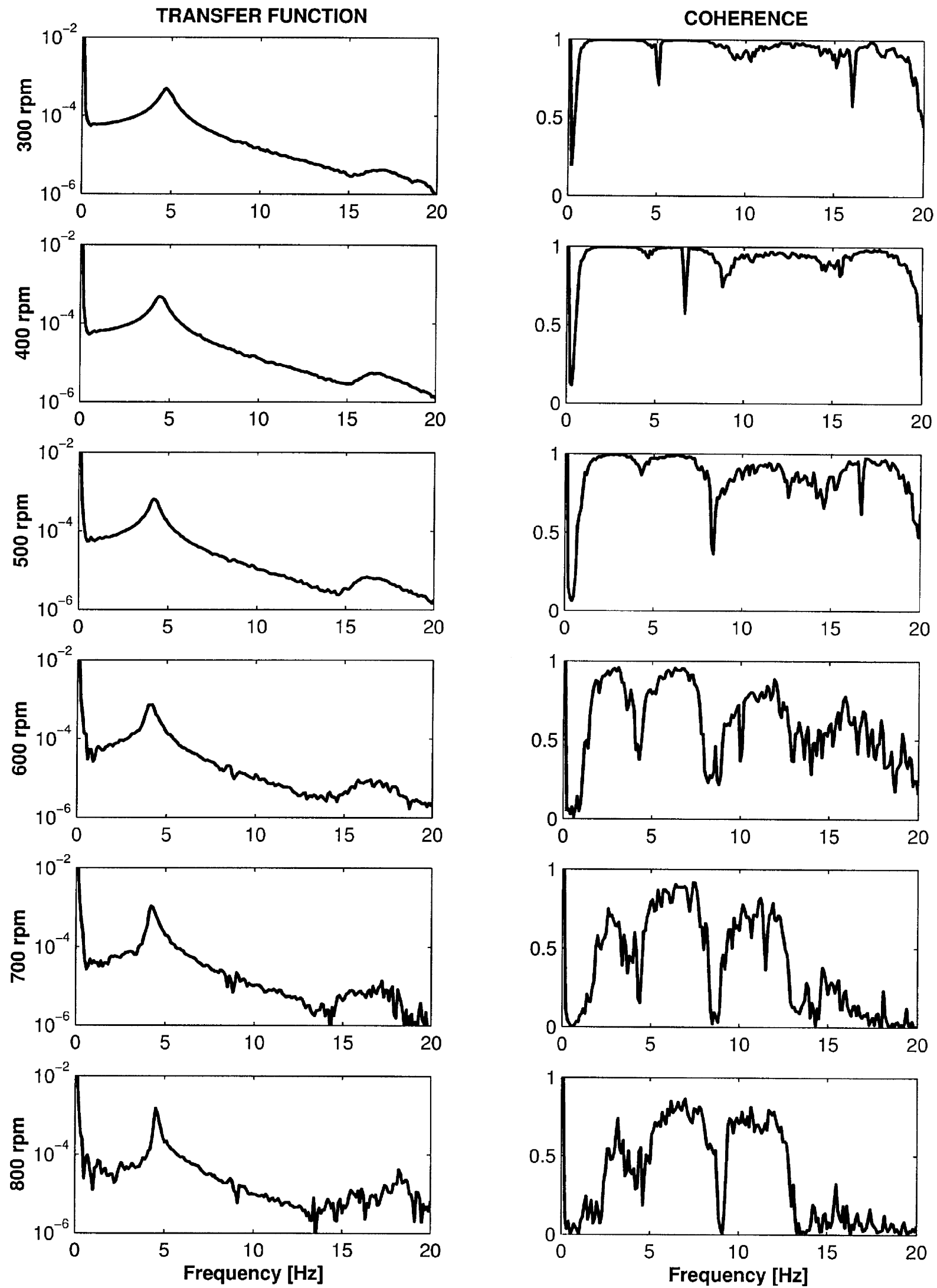

FIGURE 15 Planar configuration P2: $\varepsilon=0.5$, transfer and coherence functions (experiments). 

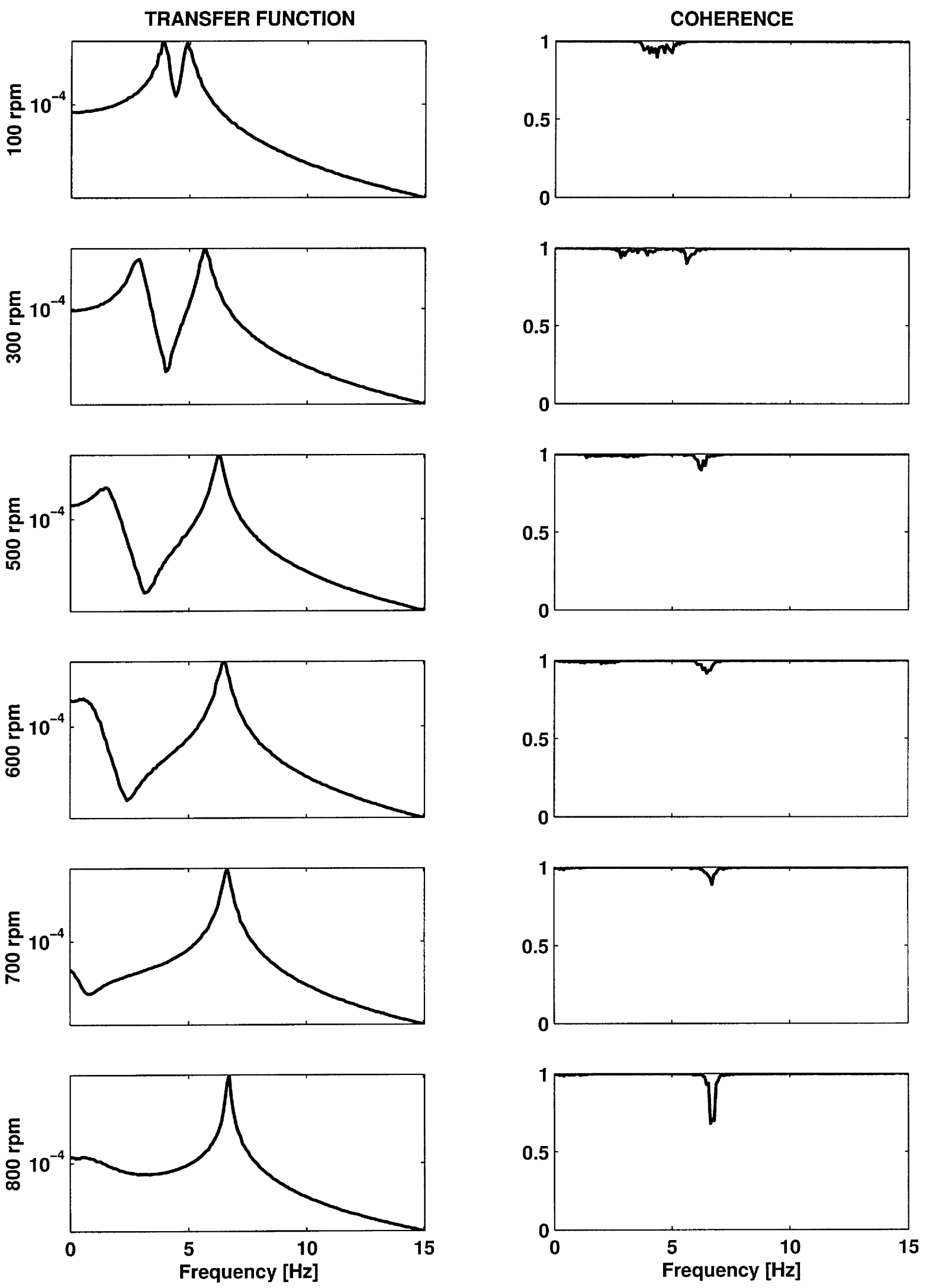

FIGURE 16 Orbital configuration O1: $\varepsilon=0$, transfer and coherence functions (nonlinear simulations). 

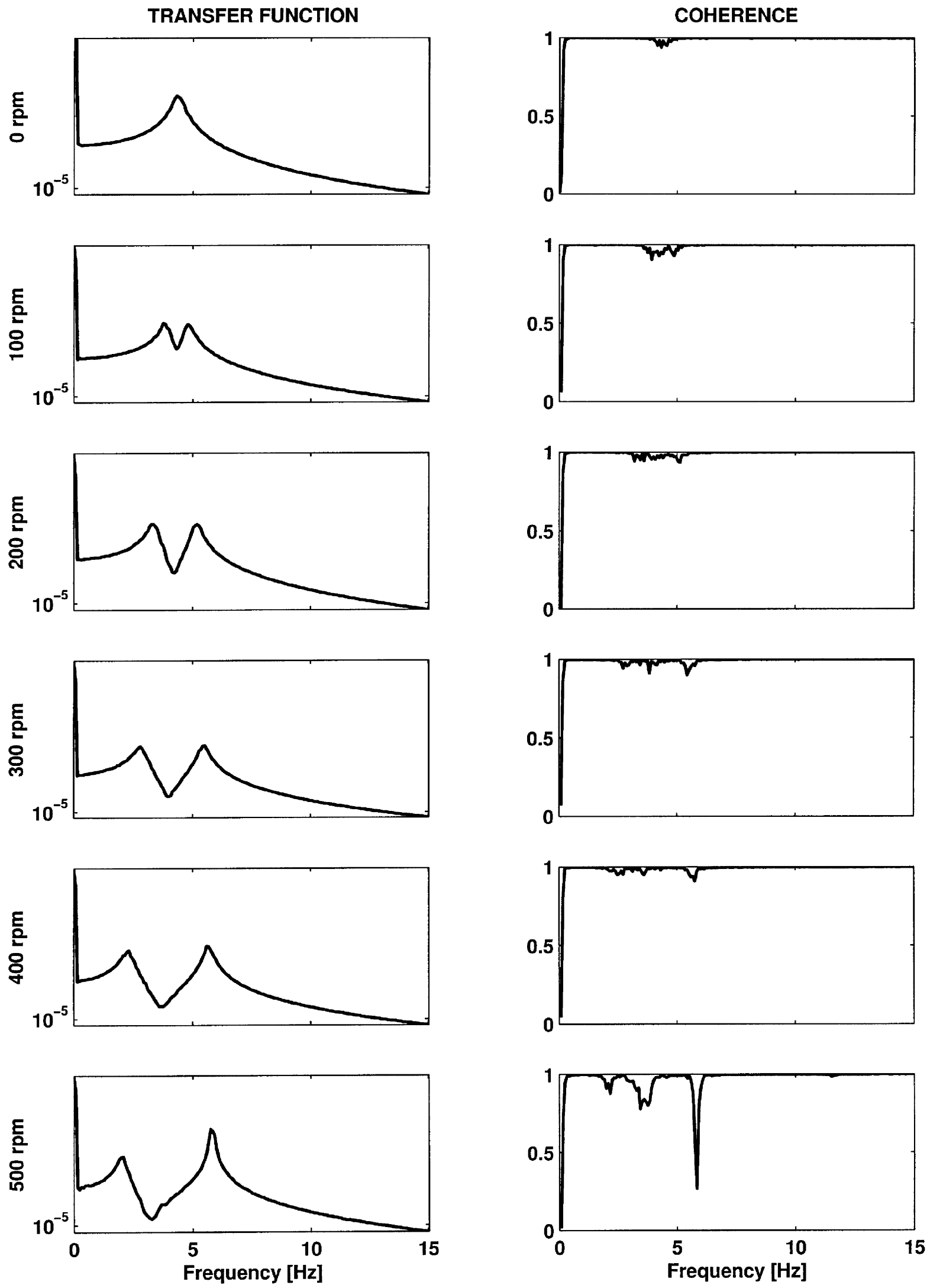

FIGURE 17 Orbital configuration O1: $\varepsilon=0.5$, transfer and coherence functions (nonlinear simulations). 


\section{CONCLUSIONS}

Besides the fact that the nonlinear model can predict the rotor dynamic eccentricity, which strongly governs the system dynamics and whose knowledge is essential for linear predictions, the present results show that:

(1) Both linear and nonlinear formulations agree with experiments when the spinning velocity and the dynamic eccentricity are low enough. In this case the system dynamics are practically linear.

(2) At higher spinning velocities the behavior of centered and eccentric rotors is somewhat different:

(a) For both planar and orbital motions, centered configurations seem to be governed mostly by linear flow effects up to the instability boundary;

(b) On the contrary, the importance of nonlinear flow forces was confirmed for eccentric configurations, well below the instability boundary. This is particularly true for planar motions. Flow nonlinearities produce a moderate stabilizing effect.

(3) It appears that post-stable whirling motion regimes (limit cycles) were also reasonably well predicted by the nonlinear flow model. Also, linear instability is predicted for the correct whirling mode.

(4) Nonlinear numerical simulations may lead to lower apparent damping values than experimentally identified. This may be due to three-dimensional flow effects not accounted for by the theoretical assumptions of the model.

From our results, it appears that the theoretical formulations for nonlinear and linearized flow forces seem to be adequate, although moderately conservative. Indeed, errors in instability predictions were found typically to be within $10 \%$, and at most $30 \%$ of the experimental observations. Therefore, in spite of some verified quantitative differences, it can be concluded that the nonlinear flow model leads to better predictions of the system dynamics.

\section{NOMENCLATURE}

$C(t) \quad$ auxiliary flow variable

$F_{X}, F_{Y} \quad$ nonlinear fluid-elastic forces

$\begin{array}{ll}F_{X}^{\text {ext }}, F_{Y}^{\text {ext }} & \text { external forces } \\ h(\theta, t) & \text { annular gap depth } \\ H & \text { average annular gap } \\ K_{s}^{X}, K_{s}^{Y} & \text { structural stiffness } \\ L & \text { rotor length } \\ M_{a} & \text { added mass: } \pi \rho R^{3} L / H \\ M_{s} & \text { structural mass } \\ p(\theta, t) & \text { gap-averaged pressure } \\ R & \text { rotor radius } \\ t & \text { time } \\ u(\theta, t) & \text { gap-averaged tangential velocity } \\ X(t), Y(t) & \text { rotor motions } \\ \gamma & \text { mass ratio: } M_{a} / M_{s} \\ \delta & \text { clearance ratio: } H / R \\ \varepsilon & \text { reduced static eccentricity } \\ \lambda_{n} & \text { eigenvalue of the flow-struc. system } \\ \theta & \text { azimuthal angle } \\ \rho & \text { fluid density } \\ \sigma_{n} & \text { real part of the eigenvalue } \lambda_{n} \\ \tau_{r}, \tau_{s} & \text { shear stresses } \\ \omega_{n} & \text { imaginary part of } \lambda_{n}\end{array}$

\section{REFERENCES}

Axisa, F. and Antunes, J. (1992) Flexural Vibrations of Rotors Immersed in Dense Fluids, Journal of Fluid and Structures, 6, 3-21.

Antunes, J., Axisa, F. and Grunenwald, T. (1996) Dynamics of Rotors Immersed in Eccentric Annular Flow: Part 1-Theory, Journal of Fluid and Structures, 10, $893-918$.

Antunes, J., Mendes, J., Moreira, M. and Grunenwald, T. (1999) A Theoretical model for Nonlinear Planar Motions of Rotors under Fluid Confinement, Journal of Fluid and Structures, 13, 103-126.

Childs, D. (1993) Turbomachinery Rotordynamics: Phenomena, Modeling and Analysis, New York, John Willey \& Sons.

Juan, J. (1994) An Eigensystem Realization Algorithm for Modal Parameter Identification and Modal Reduction, AIAA Journal of Guidance, Control and Dynamics, 8, 620-697.

Moreira, J., Antunes, J. and Pina, H., A Theoretical model for Nonlinear Orbital Motions of Rotors under Fluid Confinement, 1999 ASME Pressure Vessels and Piping Conference, 1-5 August, 1999, Boston, Massachusetts.

Moreira, M., Antunes, J. and Pina, H., An Improved Linear Model for Rotors Subject to Dissipative Annular Flows, Accepted for presentation, In: FIV 2000 (7th International Conference on Flow Induced Vibrations), 19-21 June, 2000, Lucerne, Switzerland. 

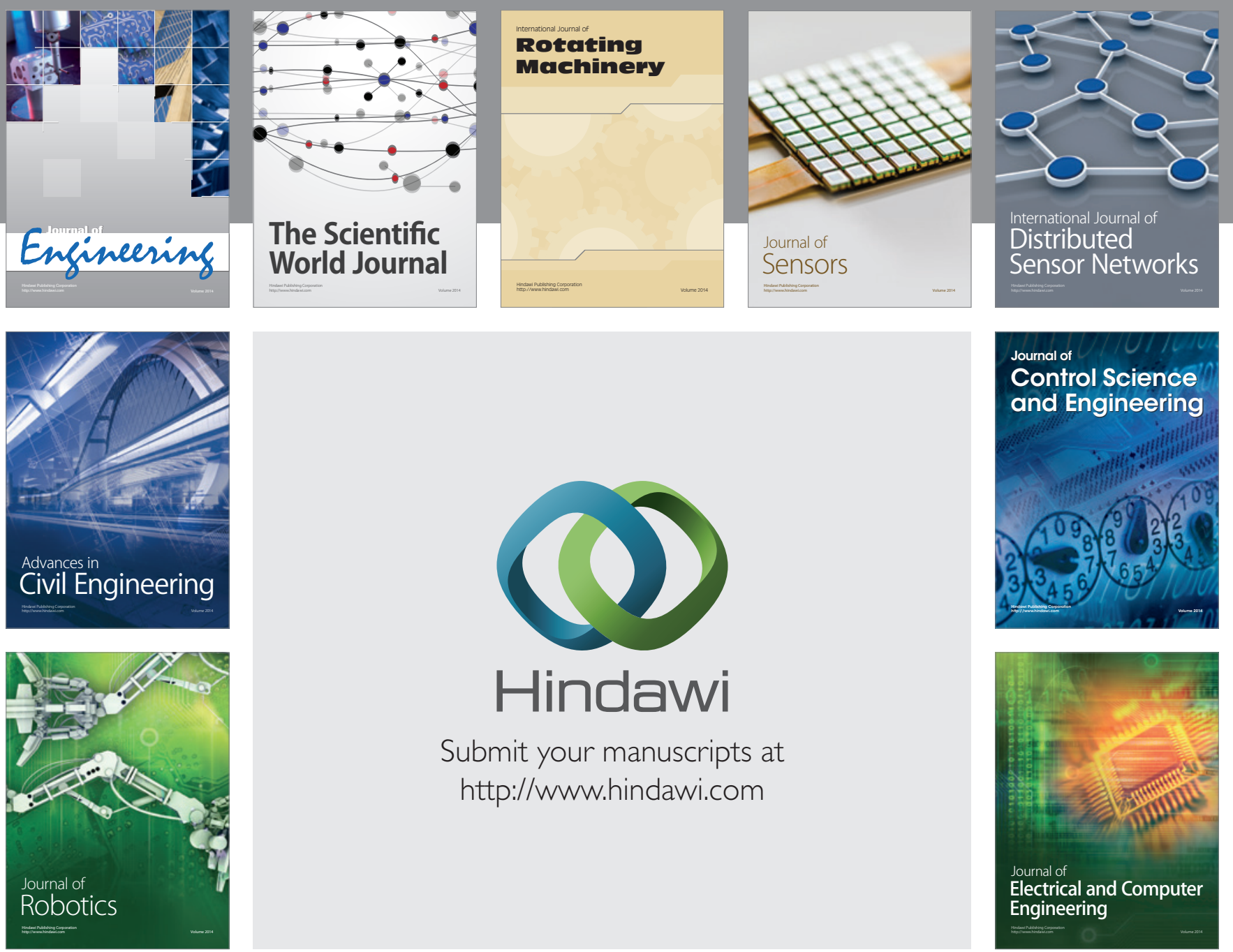

Submit your manuscripts at

http://www.hindawi.com
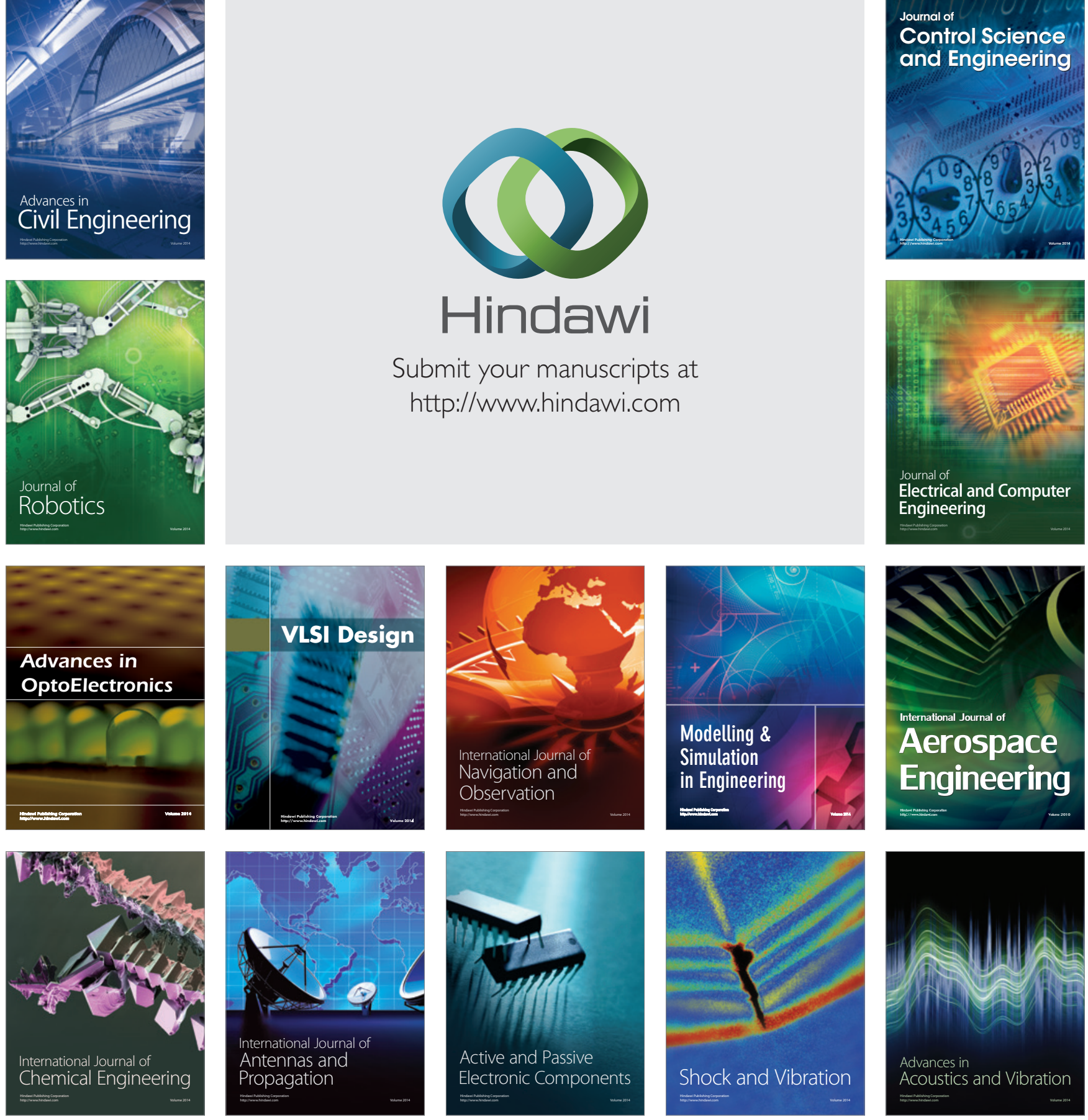